\title{
Anabases
}

ANABASES Traditions et réceptions de l'Antiquité

16 | 2012

Varia

\section{La classification des songes de Macrobe en moyen français : continuité, ruptures et déplacements}

\section{Mireille Demaules}

\section{(2) OpenEdition}

1 Journals

Édition électronique

URL : http://journals.openedition.org/anabases/3886

DOI : 10.4000/anabases.3886

ISSN : 2256-9421

Éditeur

E.R.A.S.M.E.

\section{Édition imprimée}

Date de publication : 1 octobre 2012

Pagination : 31-46

ISSN : 1774-4296

\section{Référence électronique}

Mireille Demaules, "La classification des songes de Macrobe en moyen français : continuité, ruptures et déplacements », Anabases [En ligne], 16 | 2012, mis en ligne le 01 octobre 2015, consulté le 20 octobre 2019. URL : http://journals.openedition.org/anabases/3886 ; DOI : 10.4000/anabases.3886 
Anabases 16 (2012), p. 31-46.

\section{La classification des songes de Macrobe en moyen français : continuité, ruptures et déplacements}

Mireille Demaules

Pour nommer l'expérience onirique, le français moderne dispose essentiellement de trois substantifs : le plus courant est le mot rêve, le plus littéraire est le mot songe, un peu suranné ; à côté, pour dénommer le mauvais rêve existe le substantif cauchemar. D'une certaine manière, la dénomination n'est pas très riche et nous avons coutume de ranger sous les hyperonymes songe ou rêve, selon le niveau de langue, des expériences très diverses que les Anciens distinguaient par des dénominations propres. Ainsi, il existait dans l'Antiquité grecque et latine de savantes classifications des rêves, qui ont permis de penser le rêve et sont restées valides jusqu'à l'aube de la Renaissance. L'une d'entre elles a été particulièrement célèbre au Moyen Âge, c'est celle de Macrobe, un grammairien latin de la fin du IV $\mathrm{v}^{\mathrm{e}}$ siècle - début du $\mathrm{V}^{\mathrm{e}}$ siècle après Jésus-Christ, qui a été transmise en langue latine par des traités savants, puis en langue vulgaire par des traductions dans des ouvrages didactiques. Ces transpositions en langue vernaculaire à l'usage d'un lectorat cultivé, voire savant, témoignent d'une certaine continuité dans la transmission de la classification antique des songes, mais également de ruptures ou de déplacements dans la dénomination que nous aimerions examiner, en tant qu'ils révèlent des changements dans la représentation du rêve. De plus, il faudra se demander pourquoi les termes français issus des classifications antiques ne se sont pas imposés dans la langue vulgaire. Pour finir, nous tenterons de déterminer pourquoi des mots d'origine obscure, tels que rêve et cauchemar, ont fini par s'imposer au détriment de termes reliés à la culture antique. 


\section{La classification des songes de Macrobe}

Macrobe est célèbre au Moyen Âge pour avoir écrit un commentaire mathématique, astronomique et philosophique sur un récit de rêve, composé par Cicéron dans le livre VI de son traité De re publica ${ }^{1}$. Dans ce récit, Cicéron prête à un général romain, Scipion Émilien, un rêve prémonitoire alors qu'il était venu en Afrique pour donner l'assaut à Carthage. Scipion Émilien voyait en rêve apparaître son grand-père, Scipion l'Africain, qui lui annonçait qu'il allait détruire Carthage. Outre cette prophétie, le rêve dévoile des secrets de l'univers. Scipion Émilien monte en effet dans les régions célestes d'où il contemple le monde. Son père et son grand-père l'invitent également à se tourner vers le bien pour gagner l'immortalité de l'âme. Commentant cette fable, Macrobe écrit un traité encyclopédique d'inspiration néo-platonicienne, qui initie aux sciences de la nature et à la philosophie. Pour lui, la fable du rêve est une voie d'accès au dévoilement de mystères sur l'immortalité de l'âme, que l'on ne peut approcher directement par le discours rationnel.

En préambule à son commentaire, Macrobe veut prouver la valeur de vérité du rêve auquel il accorde la même fonction que Platon attribuait au mythe : il peut révéler des vérités inaccessibles à la raison. Il entame donc une dissertation sur les songes en distinguant d'une part les songes vrais et d'autre part les songes faux ${ }^{2}$.

Parmi les songes faux, illusoires, il distingue tout d'abord l'insomnium. Le mot est un composé de la préposition in- et du mot somnium signifiant le sommeil. L'insomnium désigne ainsi la vision intérieure au sommeil et qui s'évanouit avec lui sans laisser de trace. L'insomnium est d'origine physique ou psychique. Il peut être provoqué par les besoins du corps, la faim ou la soif, ou inversement par un excès de nourriture ou de boisson. Lorsqu'il a une origine psychique, il s'agit d'un rêve provoqué par les préoccupations de la veille, les désirs, l'amour et les soucis. Par le terme visum, Macrobe désigne les visions hypnagogiques qui apparaissent entre la veille et le sommeil. Ce sont des visions confuses et désordonnées. Il classe dans cette catégorie un type de songe qu'il dénomme par un terme grec, ephialtes, et qu'il définit en se référant à une croyance populaire : il serait provoqué par un démon incube qui, pesant sur la poitrine du dormeur, produit un cauchemar oppressant.

À ces songes illusoires, Macrobe oppose ceux qui contiennent une vérité oraculaire et qui induisent chez le dormeur qui les reçoit une disposition mentale propice à la divination. L'oraculum est un songe clair, de nature programmatique : il se produit lorsqu'un parent, un ami ou une personne inspirant le respect - ce peut être un dieu ou un prêtre - révèle clairement un événement à venir ou invite à tenir une conduite de toute urgence. La visio préfigure en clair un événement qui se réalise peu de temps

1 Voir Macrobe, Commentaire au Songe de Scipion, texte établi, traduit et commenté par M. Armisen-Marchetti, 2 vol., Paris, Les Belles Lettres, 2001-2003.

2 Id., I, 3, 1-20, p. 10-16. 
après. Quant au terme somnium, il désigne le songe allégorique. Il cache sous des symboles, des voiles et des énigmes la signification du rêve qui demeure incompréhensible sans interprétation.

Cette classification des songes appelle quatre remarques.

1. Le latin somnium, qui est l'hyperonyme de toutes les catégories de songe dégagées, dérive de somnus, terme désignant le sommeil et la divinité du sommeil chez Virgile et Ovide. La forme crée un rapport étroit entre les deux mots. Indirectement, le rêve appartient au sommeil, ce qui peut être un critère permettant de le distinguer des autres expériences oniriques, telles que l'apparition ou l'extase. Le mot somnus possède des connotations négatives en latin, puisque au sens figuré il peut désigner la paresse, l'inaction, l'oisiveté, notamment chez Cicéron. Il peut aussi par métaphore évoquer la mort. Par contamination métonymique, le songe peut prendre des connotations négatives et ainsi être perçu comme une illusion. En français, somnium a donné par évolution phonétique le mot songe, mais le lien entre le songe, le sommeil, le somme n'apparaît plus si nettement qu'en latin dans la forme des mots ${ }^{3}$.

2. La catégorie du visum est assez hétérogène chez Macrobe, puisque le mot traduit deux termes grecs distincts que donne le grammairien : celui de phantasma qui désigne une apparition, une image sans consistance, et celui d'ephialtes qui dénomme un spectre induisant un songe oppresseur. Pour la catégorie de l'ephialtes, Macrobe ne produit pas de correspondant latin. La création lexicale des langues vernaculaires remédiera à ce manque dans la terminologie latine.

3. Tout autant qu'une suite d'images ou de choses vues, le songe est compris comme un message oral qui s'entend : il est un oracle, c'est-à-dire une parole prophétique. Il dit quelque chose. C'est une conception qui est commune à la Bible et à l'Antiquité gréco-latine.

4. La conception des rêves véhiculée par la typologie de Macrobe est ambiguë : elle est à la fois empreinte de magie et rationaliste. En effet elle rend compte de croyances populaires. Elle confirme le lien du songe au domaine de la magie en lui accordant une valeur oraculaire. Elle fait également référence de manière indirecte à des pratiques religieuses propres au paganisme et dont héritera la culture chrétienne. Ainsi dans la catégorie des songes insignifiants le terme ephialtes renvoie à la croyance selon laquelle des démons oppressent la poitrine du dormeur. Macrobe rapporte que selon la croyance populaire (publica persuasio), il s'agit d'un être réel. Mais lui-même attribue une origine naturelle au songe oppresseur : l'être fantastique qui agresse le rêveur entre veille et sommeil n'existe que dans son imagination. Enfin la catégorie de l'oraculum renvoie sans doute au songe homérique, mais implicitement aussi à la pratique du songe incubatoire : dans l'Antiquité, il était d'usage de dormir dans les temples

3 Voir F. Schalk, "Somnium und verwandte Wörter in den romanischen Sprachen ", in Exempla romanischer Wortgeschichte, Francfort-sur-le-Main, éd. Klostermann, 1966, p. 295-337. 
dans l'espoir d'obtenir un rêve curatif ou un rêve oraculaire qui prophétise le destin du rêveur ${ }^{4}$. Le terme oraculum désigne d'ailleurs en latin à la fois un lieu de prières, de culte, et l'oracle des dieux obtenu par un songe. Toutefois Macrobe ne développe pas cette catégorie de rêve par des exemples précis, ce qui suggère peut-être une certaine réserve de sa part. Mais le point de vue rationaliste de Macrobe ne va pas toutefois jusqu'à rejeter l'oniromancie, en particulier parce que son but est de montrer la valeur de vérité du rêve.

\section{Traduction et créations savantes en moyen français}

Le Commentaire au Songe de Scipion a été recopié et glosé durant tout le Moyen Âge mais la classification de Macrobe a été surtout commentée à partir du XII ${ }^{\mathrm{e}}$ siècle. Elle est glosée par Guillaume de Conches ${ }^{6}$ et, en dehors de la tradition des commentaires, reprise par Jean de Salisbury dans le Policraticus (vers 1159$)^{7}$, au livre II, dans les chapitres XV à XVII, et chez un auteur cistercien, identifié à Alcher de Clairvaux, dans le Liber de spiritu et anima ${ }^{8}$. Chez ces auteurs latins la terminologie macrobienne est reprise avec fidélité.

Aux XIV et $\mathrm{XV}^{\mathrm{e}}$ siècles la langue vulgaire commence à investir des champs du savoir réservés jusqu'alors à la langue latine, de sorte que l'on traduit en français des

4 On pourra se reporter sur ce point à C. A. MEIER, «Le rêve et l'incubation dans l'ancienne Grèce ", in R. Caillois et G. E. Von Grunebaum (dir.), Le rêve et les sociétés humaines, Paris, Gallimard, coll. "Bibliothèque des Sciences Humaines ", 1967, p. 290-304. Voir aussi Aelius Aristide, Discours sacrés. Rêve, religion, médecine au II siècle après J.-C., introduction et traduction par A. J. Festugiere, préface de J. Le Goff, Paris, Macula, coll. "Propylées ", 1986.

5 Sur la réception médiévale du Commentaire au Songe de Scipion, voir principalement I. Caiazzo, Lectures médiévales de Macrobe, Paris, Vrin, coll. "Études de philosophie médiévale ", 2002, p. 15-19 et p. 36. Sur sa réception dans les différentes langues vernaculaires, on se reportera également à l'ouvrage d'A. HüTTig, Macrobius im Mittelalter : ein Beitrag zur Rezeptionsgeschichte der Commentarii in Somnium Scipionis, Francfort-sur-leMain, P. Lang, 1990, "Schriften der Nationalsprachen ", p. 171-173.

6 Voir P. Dronke, Fabula, explorations into the uses of myth in medieval platonism, Leyde et Cologne, E. J. Brill, 1974, chap. 1, «Fabula : critical theories », p. 13-68. On se reportera aussi à l'excursus (p. 68-78) qui propose une sélection des gloses de Guillaume de Conches extraites de son Commentaire sur Macrobe, encore inédit dans son ensemble.

7 Jean de Salisbury, Policraticus, édition Weвb, 2 vol., Londres-Oxford, Clarendon Press, 1909, t. I, chap. XV-XVII, 429a-436a. Pour une édition plus récente, Jean de Salisbury, Policraticus (I-IV), éd. K. S. B. KeATs-Rohan, Turnhout, Brepols, Corpus Christianorum, Continuatio Mediævalis, 118, 1993, p. 94-106.

8 Alcher de Clairvaux est identifié comme le Pseudo-Augustin, auteur du Liber de spiritu et anima, Patrologie Latine, éd. Migne, t. XL, chap. XXV, col. 798. Alcher de Clairvaux reprend les cinq catégories des songes de Macrobe, mais remplace visum par le terme d'origine grecque phantasma. 
traités latins des siècles antérieurs. Ainsi le traité de Jean de Salisbury est transposé en langue vulgaire, en 1372, par un traducteur de Charles V, un frère mineur nommé Denis Foulechat, sous le titre Le Policratique ${ }^{9}$. Au milieu du XV siècle, Antoine de La Sale, précepteur des fils de Louis de Luxembourg, comte de Saint-Pol, écrit à l'usage de ses élèves un traité intitulé $L a$ Sale, une compilation de grands auteurs et d'histoires qu'il a entendues ou vécues. Au livre III, il consacre deux chapitres aux songes : l'un est une typologie des songes, visiblement calquée de Macrobe, l'autre est le récit d'un rêve célèbre du roi Crésus ${ }^{10}$. Or, il nous a semblé intéressant d'examiner comment la langue vulgaire transposait les dénominations de Macrobe. Parvenait-elle à rendre compte par une terminologie adéquate des distinctions subtiles opérées par la langue latine ? Que nous révèle de la conception du rêve la transposition en langue française ${ }^{11}$ ?

La traduction de la typologie de Macrobe en moyen français présente a priori une certaine continuité avec son original latin. En effet les traducteurs conservent les cinq catégories de rêves ainsi que la bipartition entre songes vrais et songes illusoires, laquelle donne son architecture conceptuelle à la typologie de Macrobe. Denis Foulechat débute ainsi son chapitre :

«Il sont pluseurs especes de songes et pluseurs causes et diverses figures et significacions; car ou c'est ensonge ou fantosme ou songe ou enseignement de Dieu ou vision ${ }^{12}$."

Quant à Antoine de La Sale, il introduit son exposé en ces termes :

«Il convient savoir, selon que les philosophes dient, et premier Macrobe assez près du commencement de son livre qui est nommé Disposicion de Songes, ou il dist que sont V. manieres de songes : dont la premiere est simplement appellee songe ; la seconde est appellee vision ; la tierce est appellee oracle ; la quarte est appellee insonge ; la quinte est appellee fantosme. Dont de ces.II. dernieres : de insonge et de fantosme, dit que on ne doit faire compte ${ }^{13}$."

La traduction des termes dénommant les songes vrais semble avoir été aisée tant les mots de la langue vulgaire présentent une filiation nette par les sons et le sémantisme avec leur étymon latin. Ainsi somnium est-il traduit facilement par songe et visio par vision. Le mot oracle, qui est bien le descendant transparent du mot latin oraculum et qui comme tel est conservé dans les deux textes, embarrasse néanmoins Denis Foulechat et Antoine de La Sale. Cet embarras est imputable à deux raisons. La première est purement linguistique : en moyen français le mot oracle n'évoque pas d'emblée un type de

9 Denis Foulechat, Le Policratique de Jean de Salisbury (1372), Livres I-III, éd. C. BRUCKER, Genève, Droz, 1994, Livre II, chap. XV, p. 149-152.

10 Antoine de La Sale, Euvres Complètes, éd. F. Desonay, 2 vol., Liège, Faculté de Philosophie et Lettres, Paris, Droz, 1935-1941, t. II, La Sale, p. 229-231.

11 Je remercie Jean-Marie Fritz d'avoir attiré mon attention sur ces deux textes.

12 Le Policratique, chap. XV, 1-2, p. 149.

13 La Sale, p. 229. 
rêve, mais plutôt un type d'énoncé : "une réponse divine dans l'Antiquité " ou "un lieu où la divinité rend l'oracle " selon le Dictionnaire du Moyen Français. La deuxième raison tient au rejet du paganisme, à l'impossibilité d'inclure dans la catégorie des songes vrais des rêves où apparaissent des dieux païens. Accessoirement s'ajoute le rejet de la pratique du songe incubatoire condamnée par l'Église, sans qu'elle parvienne, tout du moins jusqu'au Haut Moyen Âge, à éradiquer totalement la coutume, puisqu'on allait incuber auprès de tombeaux de saints ${ }^{14}$. La traduction du mot oraculum par oracle requiert donc une glose qui manifeste la distance du chrétien vis-à-vis des croyances païennes. Ainsi Denis Foulechat évite dans un premier temps le mot oracle et emploie pour présenter cette catégorie de rêve la périphrase : "enseignement de Dieu ${ }^{15}$." Ensuite, lorsqu'il définit plus précisément ce type de rêve, il emploie le terme oracle, mais précédé du mot espece - "espece de oracle ${ }^{16}$ "- qui marque sa réticence, puis il glose cette expression par une périphrase explicative absente de son original latin : "C'est a dire response du ciel. " L'oracle est défini comme une annonciation, une "devine volenté par bouche d'omme annunciee ${ }^{17}$ ". L'apparition des dieux anciens est rejetée comme une croyance diabolique par Jean de Salisbury et Denis Foulechat ou attribuée à une "maniere du parler de Macrobe ${ }^{18}$ " par Antoine de La Sale. Afin d'éclairer la signification de l'oracle, les auteurs médiévaux ajoutent des exemples qui illustrent la définition macrobienne et construisent sous les yeux du lecteur la traduction : ils sont principalement tirés du Nouveau Testament et prennent la forme de l'apparition d'un ange qui transmet la volonté divine à des humains. Sont cités comme oracle par exemple le songe des Rois Mages (Matthieu 2, 12), dans lequel un ange les avertit de ne pas retourner auprès d'Hérode ; ou celui de Joseph (Matthieu 2, 13), dans lequel un ange lui ordonne de fuir en Égypte avec la Vierge et le Christ nouveau-né. Hors de ces exemples, le songe oraculaire est considéré comme une superstition diabolique. Jean de Salisbury et son traducteur Denis Foulechat condamnent très expressément les rêves oraculaires de l'Énéide où apparaissent les dieux ou le défunt Anchise :

"Eneas, par l'enseigne de leur devins respons, trouva Ytalie, qui par eulz li estoit promise et de luy tres diligenment avoit esté quise et poursuye, et en ycelle, par l'inspi-

14 Voir J. Le Goff, Le christianisme et les rêves (II -VIt siècle), in L'imaginaire médiéval, Paris, Gallimard, coll. "Bibliothèque des Histoires », 1985, p. 265-316, et, plus particulièrement p. 289. On consultera également P. SAINTYVES, En marge de la légende dorée. Songes, miracles et survivances, Paris, Robert Laffont, coll. "Bouquins ", 1987 [Émile Nourry, 1930], «Des songes provoqués et de l'incubation », p. 518-523.

15 Le Policratique, 1, p. 149.

16 "Mais, quant la chose par repos esclarcist a l'annunciacion d'autrui, toutesvoies se la personne de celui qui l'annunce est honeste et honorable, ce est espece de oracle, c'est a dire response du ciel. ", Le Policratique, 42, p. 151.

17 Ibid.

18 La Sale, p. 230 : "Oracle est quant lui appert pere ou mere ou aulcune sainte personne, prestres ou aulcuns dieux, selon la maniere du parler de Macrobe. » 
racion non pas tant des esperis, que il appellent leurs dieux, comme par l'inspiracion de dyables, il establi son siege et sema la semence du lignage romain ou jardin qui mieulx leur plaisoit. Et quelle autre chose feist en songe le pere Anchises et quelle autre Jupiter et quelle Apollo et aussy les autres, qui seroit longue chose a raconter ${ }^{19}$ ?"

Ce jugement explique sans doute la disparition dans l'Eneas, adaptation médiévale (vers 1160) du texte virgilien, de tous les songes prophétiques de l'Énéide: les trois songes où apparaissent successivement à Énée Hector ${ }^{20}$, les dieux Pénates ${ }^{21}$ et Mercure $^{22}$. En revanche, l'apparition d'Anchise à Énée est conservée, peut-être parce qu'elle est conçue comme une vision éveillée ${ }^{23}$.

C'est pour la traduction des termes désignant les songes illusoires que l'on observe la tension la plus nette entre un effort de continuité, voire de fidélité, par rapport à la source latine et la nécessité de son abandon au profit d'un déplacement de la terminologie. Le mot insomnium, qui désigne le songe le plus banal, le plus quotidien chez Macrobe, est rendu par le néologisme ensonge qui est le calque de l'étymon latin. Le terme ensonge est bien attesté au XIV siècle, chez Froissart, dans le roman de Méliador (1373-1388), au sens de " rêve ", "songe ", " imagination trompeuse ": il rime avec une forme du verbe mensongier ${ }^{24}$. Il est encore attesté au XV siècle, chez Antoine de La Sale, sous la forme insonge, mais il n'est plus attesté dans le Dictionnaire de la langue française du XVI siècle d'Edmond Huguet et semble avoir disparu de la langue à cette époque. On peut supposer que ce mot n'a jamais vraiment pénétré la langue courante, en raison peut-être de sa proximité phonique avec le mot ensoigne signifiant "inquiétude ", " difficulté ".

Le mot visum n'est pas traduit par son descendant monosyllabique vis dont les sens s'étaient trop écartés de sa racine et qui signifiait principalement " avis ", " opinion ", "impression ». Il est traduit par Denis Foulechat et par Antoine de La Sale par le mot fantosme, qui a pour origine le mot grec ionien * phantagma, altéré en * phantauma dans le grec massaliote. L'emploi du mot fantosme contourne l'imprécision du mot latin visum en faisant retour au mot grec phantasma, dont il était la traduction dans le texte de Macrobe ${ }^{25}$. En moyen français, le mot fantosme désigne " une illusion ",

19 Le Policratique, chap. XV, 48-49, p. 151.

20 Virgile, Énéide, éd. et trad. de J. Perret, 3 vol., Paris, Les Belles Lettres, 1977-1980, t. I, livre II, v. 268-297, p. 48-49.

21 Id., t. I, livre III, v. 147-171, p. 80-81.

22 Id., t. I, livre IV, v. 553-570, p. 131-132.

23 Id., t. II, livre V, v. 722-739, p. 32-33. Voir Eneas, roman du XII siècle, éd. J.-J. SALVERDA de Grave, 2 vol., Paris, Champion, 1925-1929, t. I, v. 2161-2220, p. 66-68.

24 «Plus a de .X. jours que n'ö̈ / Parolle qui si bien m'agrée / Et, ou cas que je m’i recrée, / Si me di ci presentement, / De tout ton meilleur sentement, / Se tu dis voir u tu mençonges / Pour moi oster de telz ensonges. " (Jean Froissart, Meliador, éd. A. Longnon, 3 vol., Paris, Firmin-Didot, SATF, 1895-1899, t. II, v. 11867-11873, p. 74).

25 Voir Macrobe, Commentaire au Songe de Scipion, I, 3, 1, p. 10 et I, 3, 7, p. 12. 
" une chimère ", "le fruit de l'imagination " et plus spécialement " une apparition ", "un esprit malfaisant ", " un être fantomatique ». Dans tous les exemples cités par le Dictionnaire du Moyen Français, le terme est en collocation avec des mots ou des expressions qui lui donnent une signification négative, voire diabolique : illusion, euvre dyabolique, deableries. Les deux auteurs que nous avons retenus présentent le fantosme ou le songe de fantosme comme la création de l'état intermédiaire entre veille et sommeil.

Dans cette catégorie, Macrobe classait le songe oppresseur qu'il dénommait par le substantif grec ephialtes ${ }^{26}$. Les Latins ne semblent pas avoir eu de mot particulier pour désigner le cauchemar et les lettrés employaient l'expression suppressio (subp) nocturna (= "oppression nocturne »). Denis Foulechat décrit le songe oppresseur sans le nommer ni reprendre le mot grec qui se trouve pourtant dans son texte original ; il laisse à cette variété de mauvais rêve le nom de fantosme et la définit comme une maladie :

"En ceste maniere est il de une maladie dont aucuns par diverses oppressions cuide estre grevé d'aucun fais sus lui aussi comme se il feust esveillié, et cuide estre esveillié, et toutesvoies il dort, mais il repose mal ; tele puet estre dite fantosme. Et ces choses ont mieulx mestier de cure et de medecine que de nostre determinacion, et par especial quant il n'appert rien vray en eulz fors tant que ce sont tres vraies et angoisseuses passions dont personne est grevee ${ }^{27}$. "

Selon lui, le rêve oppresseur est une illusion des sens du dormeur comme l'indique l'emploi du verbe cuidier signifiant " croire à tort ", ou de la locution conjonctive " comme se ". Se fondant sur l'autorité des "phisiciens ", il donne au rêve oppresseur une cause endogène, qui peut être de nature physiologique ou psychologique. Il associe le fantosme à une condition pathologique, curable, mais néanmoins extrêmement pénible pour le rêveur. Sa compréhension du songe oppresseur est rationaliste.

Antoine de La Sale s'inscrit de la même manière dans une tradition rationaliste, mais il donne une désignation spécifique au songe oppresseur et le nomme incube :

"Aussi quant il samble que aulcune chose viengne assaillir, qui samble que monte sur luy et le charge si fort que on ne se poeut remouvoir ne parler. Et ce appellent les medecins incubz; et est une malle maladie pour tant qu'elle dure ${ }^{28}$."

Par cette définition, Antoine de La Sale se réfere à la tradition médicale de l'incube et non à la tradition théologique qui le définit comme un démon, un satyre qui agresse le rêveur, pèse sur sa poitrine et lui insuffle ainsi un rêve effrayant ${ }^{29}$. Pour Antoine de La Sale le rêve oppresseur, qui annihile la mobilité et la faculté de parole

26 Id., I, 3, 7, p. 12.

27 Le Policratique, chap. XV, 11-12, p. 149.

28 La Sale, p. 229-230.

29 M. Dulaey, Le rêve dans la vie et la pensée de saint Augustin, Paris, Études augustiniennes, 1973, "Le cauchemar ", p. 132-133. 
chez le dormeur, n'a pas de cause réelle, comme l'indique l'emploi du verbe sembler, et il est causé par un état de conscience particulier.

Au contraire de Macrobe, qui faisait allusion à la croyance populaire selon laquelle ce type de rêve est provoqué par la présence d'un être pesant sur la poitrine du dormeur, aucun des deux commentateurs ne mentionne précisément une telle croyance. De même aucun des deux ne précise l'origine de l'oppression ressentie : ils demeurent vagues. Pour Denis Foulechat il s'agit d'un poids (" fais »), tandis que selon Antoine de La Sale, l'oppression ressentie semble être due à l'agression d'" aulcune chose ". Aucun des deux ne mentionne la croyance selon laquelle le rêve effrayant serait causé par l'agression d'un animal, d'un démon ou d'une vieille femme sur la poitrine du rêveur. Pour avoir une idée des croyances que ces lettrés répugnent à mentionner, sans doute parce qu'ils y voient une résurgence du paganisme ou une superstition, il faut se reporter à l'ouvrage du médecin Bernard de Gordon, le Lilium medicine (vers 1305), dans lequel l'incube est précisément défini :

"Incubus est un fantasme qui, pendant le sommeil oppresse le corps, provoquant le mouvement et la parole. Incubus est le nom d'un démon : certains pensent que lorsque l'incube se dirige vers le corps humain - surtout si l'homme est allongé sur le dos -, par le fait d'une influence corrompue, il pèse sur le corps et fait croire à une suffocation. Lorsque cela survient aux nourrissons, ils s'étouffent souvent, parce qu'ils ne peuvent soutenir une si grande corruption : telle est l'opinion des théologiens. Mais le vulgaire pense que c'est une vieille femme qui foule et comprime le corps : il n'en est rien. Les médecins ont une meilleure opinion ${ }^{30}$."

L'exposé de Bernard de Gordon nous éclaire sur l'existence de trois interprétations différentes de l'incube. Il mentionne une interprétation théologique, sans donner de référence ni préciser la définition de l'incube élaborée par les théologiens. Selon la tradition théologique, dont on trouve la trace chez saint Augustin par exemple ${ }^{31}$, l'incube est un démon qui attaque les nourrissons pour les tuer ou les dormeurs pour avoir un commerce sexuel. Bernard de Gordon fait également état d'une croyance populaire selon laquelle il s'agit d'une vieille femme maléfique. Enfin, il rend compte de la tradition médicale, rationaliste, qui conçoit l'incube comme un simple mauvais rêve. On constate que les commentateurs de la typologie des songes de Macrobe se rattachent à

30 Bernard de Gordon, Lilium medicine, II, 24, (Lyon, G. Rouillium, 1550, p. 220-221), cité et traduit par D. JACQUART et C. THOMASSET, Sexualité et savoir médical au Moyen Âge, Paris, PUf, 1985, p. 227. Pour l'analyse de ce texte et les différentes traditions de l'incube, on se reportera à M. VAn Der LugT, Le ver, le démon et la Vierge. Les théories médiévales de la génération extraordinaire, Paris, Les Belles Lettres, coll. "L'âne d'or ", 2004, en particulier « La théorie de l'incube dans la littérature onirique et médicale », p. 317-336.

31 Augustin, De civitate Dei, XV, XXIII, 1, trad. G. CombÈs, Bibliothèque Augustinienne 36, p. 142. 
cette dernière catégorie et se refusent même à évoquer précisément les croyances qu'ils taisent. Elles demeurent du domaine du non-dit.

L'examen des traductions de la typologie de Macrobe révèle une réticence à accorder une valeur oraculaire au songe et une difficulté à concevoir l'origine du songe effrayant. Nous avons vu que sous l'espèce du fantosme, il est une sorte de pathologie. Les catégories de songes qui sont l'émanation de croyances relevant du paganisme, que ce soit l'oracle ou l'ephialtes, sont définies avec circonspection et réticence. Le terme grec ephialtes est abandonné, le terme oracle est admis avec une glose. Toutes ces réticences attestent l'émergence d'un doute sur la validité de cette typologie. Nous allons voir maintenant que la langue vulgaire courante a opéré des ruptures et des déplacements bien plus radicaux vis-à-vis de cette classification et nous essaierons d'en déterminer l'origine.

\section{Les créations de la langue vernaculaire et l'empreinte de la culture folklorique}

À partir du moyen français la langue vulgaire ne retient, parmi toutes les catégories de songe définies par Macrobe, que celles du songe et de la vision, mais sans considérer comme nécessairement vrai le phénomène onirique désigné. Les changements les plus frappants concernent la catégorie du songe illusoire, avec l'abandon de la terminologie héritée de Macrobe et l'installation dans la langue vulgaire de mots d'origine obscure qui désignent de manière adéquate les croyances folkloriques attachées à ce type de songe. On peut émettre l'hypothèse que la dénomination savante, partagée comme un trésor par le milieu clérical lettré, n'a jamais pu s'imposer en face de vocables d'origine populaire véhiculés par la masse parlante.

Le terme ensonge, insonge qui désigne le songe le plus banal, le plus quotidien, a disparu de la langue à la Renaissance. Mais s'était-il vraiment implanté dans la langue ? A-t-il jamais été populaire ? Il était usité pour dénommer le songe quotidien, sans signification. Le terme qui s'est progressivement imposé pour dénommer ce type d'expérience est le substantif rêve qui n'est attesté dans les dictionnaires qu'au $\mathrm{XVII}^{\mathrm{e}}$ siècle, postérieurement au verbe resver entré dans la langue dès le XII ${ }^{\mathrm{e}}$ siècle. En ancien français resver signifie au sens propre "errer ", " aller çà et là ", "se promener ", " rôder ", " vagabonder ". Par métaphore il a pris le sens de " divaguer ", " délirer " et avec cette signification il est souvent lié à la rime avec le verbe desver signifiant "être fou ", endesver " devenir fou ». Par extension il a pu s'appliquer à l'activité nocturne déréglée, au sens de "se livrer à une réjouissance nocturne ", "se livrer à la débauche ". Le sens moderne " avoir une vision durant le sommeil » est attesté au XIV siècle, mais ne $s^{\prime}$ 'imposera progressivement qu'au XVII ${ }^{\mathrm{e}}$ siècle. Pour bien comprendre le lien entre tous ces différents sens, qui sont dépréciatifs, il faut se reporter à l'étymologie de resver qui est sans doute liée à celle de desver. Ces deux verbes ont probablement pour racine un adjectif vagus, qui signifie " errant ", "vagabond " et qui se rattache au latin classique vagari ayant pour sens "aller çà et là ", " errer ". Jakob Jud a supposé l'existence d'un 
verbe gallo-roman *re-exvagare de même sens avec une valeur intensive ${ }^{32}$. Rêver signifie donc essentiellement "vagabonder ", " divaguer ", puis au sens figuré " vagabonder en esprit ", " délirer ». On peut donc se demander pourquoi ce mot d'origine obscure s'est progressivement imposé dans la langue vulgaire au point de remplacer le mot songe.

Il est possible que resver, puis le substantif resve, se soient imposés parce qu'ils véhiculaient un ensemble de croyances très anciennes et largement répandues selon lesquelles la nuit, durant le sommeil, l'âme ou l'esprit se détache du corps pour voyager, vagabonder ${ }^{33}$. Cette croyance est bien attestée par des légendes recueillies au Moyen Âge et dans des contes populaires parvenus jusqu'à nos jours. Au Moyen Âge, dans la deuxième partie du Roman de la Rose (écrite vers 1270-1280), Jean de Meun expose et réfute la légende de dame Habonde. Cette réfutation s'inscrit dans le discours de Nature qui rapporte que maintes gens s'imaginent devenir la nuit des " estries " (v. 18396), c'est-à-dire des stryges, des vampires ou des sorcières, qui errent la nuit en compagnie de dame Habonde. De vieilles femmes prétendent que le tiers de l'humanité possède cette faculté de quitter le corps, trois fois la semaine, durant le sommeil, pour accompagner les "bonnes dames " et pénétrer avec elles dans les maisons à travers les fentes, les chatières ou les crevasses. Au réveil, l'âme qui a couru le monde durant la nuit réintègre le corps, mais si celui-ci a été retourné, elle ne peut plus rentrer en lui ${ }^{34}$. Les «bonnes dames » et " dame Habonde » en particulier sont des femmes surnaturelles, dotées de la faculté de voler dans les airs. Dans les maisons qu'elle visite, et où elle trouve des offrandes sous forme de quelque boisson ou nourriture, dame Habonde apporte l'abondance de biens. Selon Nature, qui est le porte-parole du rationaliste Jean de Meun, cette légende est une "folie horrible " (v. 18419), des " fables " (v. 18446) véhiculées par de " foles vielles " (v. 18459). Toutefois, même s’il lui dénie une quelconque vérité, Jean de Meun transmet et fait rayonner malgré lui cette légende qui semble bien accréditée au XIII ${ }^{\mathrm{e}}$ siècle.

Par ailleurs, il existe un conte type $(1645$ A) répertorié par A. Aarne et S. Thompson sous le titre Dream of Treasure Bought, qui témoigne de la croyance au vagabondage de l'âme durant le sommeil ${ }^{35}$. Ce conte met en scène deux personnages,

32 Sur cette étymologie de resver, rêver, voir J. Jud, "Rêver et Desver ", Romania, t. LXII, 1936, p. 145-157. Pour une synthèse des hypothèses étymologiques, on se reportera à l'article "rêver " du Dictionnaire historique de la langue française, établi sous la direction d'A. Rey, 2 vol., Paris, Dictionnaires Le Robert, 1992, t. II, p. 1797-1798.

33 Sur ce point voir l'article de D. FABRE, "Rêver, le mot, la chose, l'histoire ", Terrain 26 (mars 1996), p. 69-82.

34 Guillaume de Lorris et Jean de Meun, Le Roman de la Rose, éd. F. LeCoy, 3 vol., Paris, Champion, CFMA, 1965-1970, t. III, v. 18395-18484, p. 52-55. Sur ce passage du Roman de la Rose, nous nous permettons de renvoyer à notre ouvrage, La Corne et l'Ivoire. Étude sur le récit de rêve dans la littérature romanesque des XII et XIII siècles, Paris, Champion, coll. "Nouvelle Bibliothèque du Moyen Âge ", 2010, p. 583-590.

35 A. Aarne et S. Thompson, The types of the folktale: a classification and bibliography, Folklore fellows communications, $\mathrm{n}^{\circ} 184$, Helsinki, 1961, "Dream of Treasure Bought. 
un rêveur et un observateur. Tandis que l'un est endormi, l'autre aperçoit sortant de sa bouche un petit animal : un lézard, une mouche, une souris, un serpenteau, qui cherche à franchir un ruisseau, visite le crâne d'un cheval, d'un âne ou s'enfonce dans un trou de la montagne. L'animal réintègre ensuite le corps endormi et le dormeur s'éveille aussitôt : il raconte qu'en rêve il a visité un palais et découvert un trésor. Le rêveur et l'observateur confrontent leurs points de vue et, creusant la terre sous le crâne de l'animal, découvrent un trésor ${ }^{36}$. Une célèbre version de ce récit est relatée par Paul Diacre dans l'Histoire des Lombards qui met en scène le roi Gontran de Bourgogne dans le rôle du rêveur ${ }^{37}$, une autre est recueillie lors de l'interrogatoire du berger Pierre Maury par l'inquisiteur Jacques Fournier et relatée par Emmanuel Le Roy Ladurie dans Montaillou, village occitan de 1294 à 1324³8, enfin Félix Arnaudin dans les Contes populaires de la Grande-Lande la recueille en 1881 sous le titre Lou rèbe ${ }^{39}$. Ce conte, dans lequel l'animal représente l'âme ou l'esprit du rêveur qui s'échappe de son corps à l'occasion du rêve, reflète une représentation du rêve comme vagabondage, voyage de l'esprit ou de l'âme en dehors de son enveloppe charnelle. Par son étymologie supposée, le mot " rêve " s'accorde ainsi avec des croyances très anciennes refoulées par la culture savante mais bien présentes dans le folklore, de sorte qu'il se serait peut-être imposé dans la langue sous la pression d'un usage populaire. Ce phénomène pourrait expliquer pourquoi, alors que le substantif "rêve » n'est attesté pour la première fois qu'au XVII ${ }^{\mathrm{e}}$ siècle, il est noté inexplicablement comme "vieux " par Antoine Furetière

Treasure has been seen by man's soul absent in sleep in form of fly (or bubble). The purchaser of the dream finds the treasure." (p. 469).

36 Voir D. FABRE, « Rêver, le mot, la chose, l'histoire », p. 72-74.

37 C. Ginzburg rapporte l'histoire dans Le sabbat des sorcières, Paris, Gallimard, coll. "Bibliothèque des histoires ", 1992, p. 147 : «L'historien lombard Paul le Diacre, qui vécut au VIII siècle, raconte que, un jour, de la bouche du roi burgonde Guntram, qui dormait veillé par un écuyer, sortit soudain un animal, une sorte de petit serpent. Celui-ci se dirigea vers un ruisseau non loin de là, qu'il tenta en vain de traverser. Alors l'écuyer posa son épée en travers, d'une rive à l'autre. Le serpent passa de l'autre côté et disparut derrière une colline; quelque temps plus tard, il fit le même chemin à l'envers, et se glissa dans la bouche du dormeur. Le roi s'éveilla et dit qu'il avait rêvé qu'il passait sur un pont de fer, pour entrer ensuite dans une montagne où était caché un trésor (qui fut effectivement trouvé). »

38 E. Le Roy Ladurie, Montaillou, village occitan de 1294 à 1324, Paris, Gallimard, coll. "Bibliothèque des Histoires », 1982 [1 ${ }^{\text {re }}$ éd. 1975], p. 608-609. Dans cette version, c'est un lézard qui sort de la bouche du dormeur et passe une rivière sur une planche avant d'aller visiter le crâne d'un âne. L'observateur ôte la planche, de sorte que le lézard ne pouvant plus passer la rivière, le corps du dormeur s'agite énormément. Le veilleur ayant remis la planche, le lézard peut traverser la rivière et réintégrer le corps du dormeur en passant par la bouche. Le parfait cathare qui interprète l'aventure déclare que l'âme demeure attachée au corps, mais que l'esprit s'évade et vagabonde en rêve.

39 F. Arnaudin, Contes populaires de la Grande-Lande, éd. J. Boisgontier et G. Latry, Bordeaux, Confluences, 1994, p. 276-277. En l'occurrence l'animal qui s'échappe de la bouche du rêveur est une mouche. 
et commenté en ces termes dans la première édition du Dictionnaire de l'Académie Française (1694) : "Il est de peu d'usage et bas. " Il est "vieux " et " bas " peut-être parce qu'il véhicule un ensemble de croyances très anciennes, et " de peu d'usage " parce qu'il n'est pas généralisé dans la culture savante écrite, ce qui ne signifie pas qu'il ne l'était pas dans la culture orale.

On retrouverait un phénomène de rupture et de déplacement similaires pour la catégorie définie par Macrobe sous le terme de visum. Le mot fantosme qui le traduit dans les classifications des XIV et $\mathrm{XV}^{\mathrm{e}}$ siècles s'est spécialisé dans la langue savante tout d'abord pour désigner les spectres et les apparitions de défunts. Dans la langue vulgaire, il existait pour dénommer le cauchemar le substantif masculin apesart, dérivé du verbe peser et qui exprime la sensation d'oppression que ressent le rêveur. Mais ce terme relevé, selon le dictionnaire d'ancien français de Tobler et Lommatzsch, au XIV siècle chez Raoul de Presles, ne semble pas avoir connu une large diffusion : il n'est pas attesté dans le Dictionnaire du Moyen Français. Le substantif incube (1372), calque savant du latin incubus, est resté un terme de théologie désignant un démon masculin censé abuser des femmes durant leur sommeil, par opposition à succube désignant un démon féminin. Le terme d'ancien français enquibedes, résultat probable d'un croisement entre incubus et equipedes (" pieds de cheval »), est usité vers 1200-1210 dans le Roman de Merlin de Robert de Boron pour désigner la variété de démon aérien, à laquelle appartient le père de Merlin :

"Je voil [dit Merlin] que tu saiches et croies que je sui filz d'un enemi qui engigna ma mere, et cele meniere d'enemi qui me conçut a non enquibedes et sont et repairent en l'air ${ }^{40}$."

Ce mot, qui ne bénéficie d'aucune entrée ni dans le dictionnaire de ToblerLommatzsch ni dans celui de Godefroy, est un hapax plaisant, qui, selon Anne Berthelot, " a l'avantage de laisser affleurer les origines mythiques de ces créatures, proches des "chèvre-pieds" ou satyres de l'Antiquité, réputés pour leur luxure ${ }^{41}$ ». Mais il reste une création de clerc. Le substantif qui s'impose pour désigner le rêve oppressant est un mot de la langue rustique, d'origine picarde : le mot cauchemar. Précisons que, si l'étymologie de resver est obscure, celle de cauchemar est elle aussi assez embrouillée, ce qui témoigne d'une origine orale se perdant dans la nuit des temps. Ce terme est tout d'abord attesté sous la forme cauquemare (1375), puis cauchemare (1584), avant de se fixer en cauchemar (1677). Il a des variantes régionales recueillies

40 Robert de Boron, Merlin, roman du XIII siècle, éd. A. MichA, Paris-Genève, Droz, 1980, 15, 20, p. 68.

41 Merlin, texte établi par I. Freire-Nunes, présenté par P. WaLter, traduit et annoté par A. Berthelot, dans Le Livre du Graal, 3 vol., Paris, Gallimard, coll. «Bibliothèque de la Pléiade ", 2001-2009, t. I, p. 1774, note 3. Pour le terme enquibedes, le manuscrit S 526 de la Bibliothèque Universitaire de Bonn, sur lequel est fondée l'édition de la Pléiade, présente la variante esquibedes, traduit par « équipède " (voir p. 606, \$34). 
dans le Grand dictionnaire universel du XIX siècle de Pierre Larousse et le Trésor de la Langue Française, parmi lesquelles on peut citer les termes cauquevieille (lyonnais), chauchi-vieilli (Isère), chauce-vieille (Rhône), chaouce-vielio (Languedoc). Le premier élément du mot (cauche) est une forme verbale de cauchier, résultant d'un croisement entre le picard cauquier et l'ancien français chauchier signifiant " presser ", "fouler aux pieds ", " piétiner ". L'origine de ces verbes est probablement le latin calcare de même sens. La seconde partie du mot est l'ancien picard mare, emprunté au moyen néerlandais mare, "fantôme qui provoque le cauchemar ». Ce mot néerlandais est attesté dans toutes les langues d'origine germanique : il correspond à l'anglais mare "spectre " qui entre dans la composition du mot nightmare, à l'ancien haut allemand mar et mara et au moyen haut allemand mar, à l'allemand moderne Marh, au norrois mara. L'origine du deuxième constituant du mot est encore discutée par les étymologistes, mais l'hypothèse soutenue par Claude Lecouteux est que le mot mar remonterait à une racine indo-européenne * mer signifiant " mourir ${ }^{42}$ ». Cette étymologie correspond bien à la description du "songe de fantôme " : c'est un spectre malfaisant qui revient hanter le vivant. À partir de cette origine sémantique, qui correspond sans doute à la croyance originelle, on peut repérer une superposition de croyances d'origine culturelle parfois différente (latine, autochtone), ce dont témoigne par exemple au début du XIII ${ }^{\mathrm{e}}$ siècle le livre III des Otia Imperialia de Gervais de Tilbury, qui rapporte plusieurs légendes au sujet du cauchemar. Selon certaines croyances, l'être irréel qui agresse le rêveur est un démon ou encore, une lamie, monstre fabuleux qui passait pour dévorer les enfants :

"Les lamies, appelées communément «masques » ou " estries » en français, sont, aux dires des médecins, des visions nocturnes qui, du fait de l'épaississement des humeurs, troublent les âmes des dormeurs et les écrasent de leur poids.

Augustin d'autre part dit, en citant certains auteurs, que ce sont des démons qui, à partir d'âmes malfaisantes, emplissent des corps aériens ${ }^{43}$."

Gervais mentionne une autre croyance qui comprend les démons oppresseurs comme une catégorie d'êtres humains - des sorciers et des sorcières - qui tourmentent les dormeurs. Il s'agit là d'une anthropomorphisation de la croyance :

"Mais pour ne pas heurter les habitudes et les oreilles des hommes, posons que c'est le destin fatal de certaines femmes et certains hommes qu'ils parcourent la nuit les pays d'un vol très rapide, pénètrent dans les maisons, tourmentent les dormeurs, leur donnent des songes lourds qui les font pleurer : on les voit aussi manger, allumer les lumières,

42 C. Lecouteux, «Mara-Ephialtes-Incubus. Le Cauchemar chez les Peuples germaniques », Études germaniques, $42^{\mathrm{e}}$ année, janvier-mars 1987, p. 1-24, et plus particulièrement p. 5.

43 Le Livre des merveilles : divertissement pour un empereur (troisième partie) de Gervais de Tilbury, trad. fr. A. Duchesne, Paris, Les Belles Lettres, coll. "La Roue à Livres », 1992, 86, p. 94. 
disjoindre les os des hommes pour les remettre parfois dans un autre ordre, boire le sang humain et déplacer les petits enfants d'un endroit à l'autre ${ }^{44}$."

On observe ainsi une évolution de la croyance qui fait de l'être irréel qui oppresse le rêveur un démon, puis un être humain maléfique, le plus souvent une vieille femme, doté de pouvoirs surnaturels. À ces croyances s'oppose la doctrine des médecins qui attribuent le cauchemar à un simple épaississement des humeurs. Ces déplacements dans les croyances s'observent dans la polysémie du mot cauquemare, qui signifie à la fin du Moyen Âge " être féerique ", " démon de la nuit " et "sorcière ". Dans les Évangiles des Quenouilles ${ }^{45}$, recueil de propos échangés à la veillée par des matrones, entre Noël et la Chandeleur, écrit dans la seconde moitié du XV siècle, la cauquemare est un être fantastique de la nuit, associé au lutin et au loup-garou, qui vient tourmenter l'homme durant son sommeil ${ }^{46}$. À l'articulation des $\mathrm{XV}^{\mathrm{e}}$ et $\mathrm{XVI} \mathrm{I}^{\mathrm{e}}$ siècles, chez le poète Jean Lemaire de Belges (1474-ca 1525), le mot cauquemare a pour sens "succube " en collocation avec luiton, et peut-être "sorcière " lorsqu'il est précédé de l'adjectif "vieille ", comme dans ces exemples cités par Le Dictionnaire de la langue française du XVI siècle de Huguet:

- « [Le diamant] chasse ces fantosmes nocturnes, qu'on appelle Incubes et Succubes. Ce sont Luittons et Cauquemares, et dissipe toutes vanitez de songes. " (La Couronne Margaritique, IV, 76);

- "Griffons hideux, qui mengent gens barbares ; Fiers Loups garoux, et vieilles Cauquemares. " (2e Epistre de l'Amand Verd, III, 25).

$\mathrm{Au} \mathrm{XVI}{ }^{\mathrm{e}}$ siècle, dans le traité De la démonomanie des sorciers de Jean Bodin (1580), la cauquemare est la face nocturne de la sorcière :

"Au pays de Valois et de Pycardie, il y a une sorte de sorcieres qu'ils appellent cochemares, et de faict Nicolas Noblet, riche laboureur demeurant a Hautefontaine en Valois, m'a dit que luy estant jeune garçon il sentoit souvent la nuict tels incubes, ou ephialtes,

44 Ibid., p. 94-95.

45 M. Jeay, Les Évangiles des Quenouilles, Montréal, Presses de l'Université de Montréal et Paris, Vrin, 1985. Voir la traduction d'A. Paupert, in Voix de femmes au Moyen Âge. Savoir, mystique, poésie, amour, sorcellerie XII $-X V^{e}$ siècle, Paris, Robert Laffont, coll. "Bouquins ", 2006, p. 727-817. Du même auteur, on lira Les fileuses et le clerc. Une étude sur les Évangiles des Quenouilles, Paris, Champion, 1990. Voir aussi P. UHL, "De la "cauquemare" et du "luiton". Le témoignage complémentaire des Évangiles des Quenouilles et de Perceforest", in Le Cauchemar, mythologie, folklore, arts et littérature, textes réunis par B. Terramorsi, Paris, Sedes-Le Publieur, 2003, p. 87-107.

46 Voir M. JeaY, Les Évangiles des Quenouilles, p. 143: "Se un homme a tele destinee d'estre leu warou, c'est fort se son filz n'en tient. Et se fille a et nulz filz, volentiers sont quauquemaires. " La quauquemaire peut descendre d'un loup-garou. Il existe ainsi une parenté des esprits malfaisants. 
qu'il appelloit cochemares, et le jour suyvant au matin la vieille sorciere, qu'il craignoit, ne failloit point a venir querir du feu ou autre chose, quand la nuit cela luy estoit advenu. » (Demon., 108, vo,$[\mathrm{G}]$ ).

Dans cet exemple, le mot cochemare fonctionne comme une réalisation dialectale de l'hyperonyme sorcière. Ce sens semble s'imposer au moment de la répression de la sorcellerie. Parce qu'il était d'usage courant, et que son origine rustique lui permettait d'agréger des sémantismes divers, reflétant des croyances populaires, le terme cauquemare, cauchemar a ainsi remplacé les mots savants incube, calque du latin, et fantosme, d'origine grecque. Ce sont donc des mots "sans pedigree ", selon l'heureuse expression de Daniel Fabre définissant le mot rêve ${ }^{47}$, au sémantisme lourd de croyances populaires qui ont remplacé les termes issus de la classification des songes de Macrobe, restée l'apanage lexicologique d'une élite lettrée.

Si l'on observe le devenir de la classification des songes de Macrobe au Moyen Âge, on remarque tout d'abord une diffusion large dans les milieux lettrés jusqu'au $\mathrm{Xv}^{\mathrm{e}}$ siècle, comme en témoignent les traductions en langue vulgaire. On mesure la validité de cette classification dans les esprits à la création lexicale du terme ensonge, censé rendre le plus fidèlement possible la catégorie de l'insomnium. Toutefois, l'examen comparatif de deux textes rédigés au Xve siècle, l'un relevant de la culture savante, $L a$ Sale, l'autre de la langue populaire, Les Évangiles des Quenouilles, révèle que la typologie savante n'a pas pénétré la langue vulgaire commune, en particulier pour dénommer les variétés du songe illusoire. Les mots qui s'imposent à partir du moyen français puis du français classique sont des mots d'origine obscure, sans doute rustique, qui véhiculent des croyances populaires, au rang desquelles il faut placer celle de la migration de l'âme et celle de l'oniropompie, pouvoir qu'ont certaines créatures, démons ou sorcières, d'insuffler des rêves. L'étude du devenir de la terminologie macrobienne des songes démontre que l'histoire de la vie des mots s'explique parfois grâce au croisement de données mythologiques et linguistiques.

Mireille Demaules

Université d'Artois (pôle d'Arras)

Centre "Textes et Cultures" EA 4028

mireille.demaules@wanadoo.fr

47 D. FABRE : « [...] Rêve était vraiment un mot sans pedigree, peut-être sorti tout cru du parler ordinaire» ("Rêver, le mot, la chose, l'histoire », p. 70). Cette remarque vaut également pour cauchemar, c'est ce que nous avons voulu montrer. 